\title{
Combination therapy in cholesterol reduction: focus on ezetimibe and statins
}

\author{
Liliana Grigore' \\ Giuseppe Danilo Norata ${ }^{1,2}$ \\ Alberico L Catapano ${ }^{1,2}$ \\ 'Department of Pharmacological \\ Sciences, University of Milan, Milan, \\ Italy; ${ }^{2}$ Center for the Study of \\ Atherosclerosis, Bassini Hospital, \\ Cinisello B, Italy
}

\begin{abstract}
Although widely used in lipid lowering therapy, HMG CoA reductase inhibitors (even when administered at high doses) are frequently insufficient to achieve guideline-recommended LDL-C goals for many patients with hypercholesterolemia in everyday clinical practice. Many patients do not achieve LDL-C goal on the initial dose of statin and the majority of these patients does not reach their goal after 6 months. As a consequence, a wide therapeutic gap exists between target LDL-C levels and those typically achieved in clinical practice. A recent and more effective therapeutic hypocholesterolemic strategy is to treat the two main sources of cholesterol simultaneously (production of cholesterol, mainly in the liver, and absorption of cholesterol in the intestine) with a complementary mechanism of action, by co-administering ezetimibe, a novel agent inhibiting cholesterol absorption, with a statin, which inhibits cholesterol production in the liver. Ezetimibe can be effectively and safely co-administered with any dose of any statin and, compared with the single inhibition of cholesterol production, afforded by statins alone, provides consistently greater reductions in LDL-C through dual inhibition of both cholesterol production and absorption. We summarize the pivotal role of both the liver and intestine in the overall balance of cholesterol in the body and describe the clinical impact and relevance of using ezetimibe either alone or co-administered with statins in controlling elevated levels of plasma LDL cholesterol.
\end{abstract}

Keywords: hypercholesterolemia, cholesterol absorption, cholesterol biosynthesis, ezetimibe, HMG CoA reductase inhibitors, LDL-C

\section{Introduction}

Hypercholesterolemia plays a key role in the development and progression of atherosclerosis and is a proven risk factor for coronary heart disease (CHD) (Wilson 1980; Stamler 1986; Steinberg 1990; Castelli 1992). Therapeutic interventions aimed at lowering cholesterol levels both in primary and secondary prevention show a clear reduction in the incidence of CHD and stroke (Anon 1984; Manninen et al 1988; Frick et al 1987; Anon 1994; Sheperd et al 1995; Downs et al 2001; Collins 2004).

Although widely used in lipid lowering therapy, HMG CoA reductase inhibitors (even when administered at high doses) are frequently insufficient to achieve guidelinerecommended low-density lipoprotein cholesterol (LDL-C) goals for many patients with hypercholesterolemia in everyday clinical practice (Goettsch et al 2004). According to a recent study, a large proportion of high-risk hyperlipidemic patients receiving statins alone are not at goal even when physicians were free to use any statin and titrate according to their professional judgment (Foley et al 2003). Over half (52\%) of patients did not achieve LDL-C goal on the initial dose of statin, and $86 \%$ of these patients had still not reached goal after 6 months (Foley et al 2003). Thus, a wide therapeutic gap exists between target LDL-C levels and what is achieved in clinical practice (Neal et al 2003).

The therapeutic gap will undoubtedly increase in light of the recent amendments of the National Cholesterol Education Program Adult Treatment Program III (NCEP 
ATP III) guidelines, which recommend even more aggressive reductions in LDL-C levels for patients at high risk of CHD (Grundy et al 2004). The more aggressive cholesterol treatment goals proposed by the revised guidelines call for a more effective approach to maximize the cardiovascular benefits associated with lower LDL-C levels.

In the human body there are two major sources of cholesterol: first, the gastrointestinal tract where daily cholesterol is derived from the diet, bile input and desquamated cells (Levy et al 2007); second, the liver which is the major source of cholesterol synthesis; in the human body (Goldstein et al 1990). Approximately 50\% of the cholesterol pool is absorbed and recirculated through the intestine, while the remainder is excreted through the feces (Lammert et al 2005; Wang et al 2007). The intestinal pool is composed of both dietary, and the majority, from biliary excretion. A recent and more effective therapeutic strategy, is to treat both sources of cholesterol simultaneously with a complementary mechanism of action, by co-administering ezetimibe, a novel agent inhibiting cholesterol absorption, together with a statin, which inhibits cholesterol production in the liver (Lammert et al 2005; Levy et al 2007; Wang et al 2007). This results in dual inhibition of both sources of cholesterol provides significantly greater LDL-C reduction and subsequent goal attainment.

Ezetimibe can be effectively co-administered with any dose of any statin; indeed the benefits are consistent across the statin brand and dose subgroups and compared with single inhibition of cholesterol production, afforded by statins alone, provides consistently greater reductions in LDL-C through dual inhibition of both cholesterol production and absorption (Gagne et al 2002; Ballantyne et al 2003; Kerzner et al 2003; Melani et al 2003; ) The single product of ezetimibe/ simvastatin provides superior LDL-C lowering efficacy with improved LDL-C goal attainment (Ballantyne et al 2004; Feldman et al 2004; Ballantyne et al 2005).

Here we summarize the pivotal role of both the liver and intestine in the overall balance of cholesterol in the body and describe the clinical impact and relevance of inhibiting both sources of cholesterol.

\section{The liver and intestine: two sources of cholesterol}

The average adult body contains approximately $140 \mathrm{~g}$ of sterols, mainly in the form of cholesterol (Cook et al 1958). This pool of cholesterol is derived from two major sources: synthesis of cholesterol by the liver (and extrahepatic sites) and absorption by the intestines (Goldstein et al 1990; Levy et al 2007). Approximately 500-1400 mg/day of cholesterol enters the body's cholesterol pool from synthesis (500-1000 $\mathrm{mg}$ ) and diet (up to $500 \mathrm{mg} /$ day) (Cook et al 1958; Illingworth et al 1995). However, the cholesterol pool usually changes little because cholesterol input is approximately balanced by cholesterol output via excretion in bile/feces, skin excretion, steroid hormone synthesis, etc (Cook et al 1958; Illingworth et al 1995).

The liver plays a central role in balancing cholesterol from all sources and also plays pivotal role in regulating plasma LDL-C levels (Dietschy et al 1993). The hepatic pool of cholesterol is derived from local biosynthesis and from chylomicron remnants and lipoproteins. Cholesterol biosynthesis is regulated by the rate-limiting enzyme HMG CoA reductase, which catalyzes production of mevalonic acid from HMG CoA and represents the therapeutic target for statins. Cholesterol produced by liver is either secreted in bile or incorporated into lipoproteins (mainly VLDL) and secreted into plasma.

Intestinal cholesterol absorption represents another major route for the entry of cholesterol into the body, and, thus, this source can influence LDL-C concentration (Wang et al 2007). Daily the cholesterol pool in the intestine comes from either dietary cholesterol (300-500 mg), from biliary excretion $(800-1200 \mathrm{mg})$ and from desquamated cells $(300 \mathrm{mg})$ (Lammert et al 2005; Wang et al 2007). Of note, as a large part of the cholesterol absorbed results from the reabsorbtion of cholesterol excreted through the bile, it is appropriate to talk about entero-hepatic recirculation). The excess cholesterol is then excreted through the feces. Although the details of the molecular transport of cholesterol absorption are not fully understood, it is clear that cholesterol absorption in the intestine involves a complex triphasic process (Figure 1): intraluminal phase (digestion/hydrolysis of dietary lipids and micellar solubilization of cholesterol), membrane transport phase (cholesterol release from micelles at brush border membrane and uptake into enterocytes), and an intracellular phase (re-esterification, incorporation into nascent chylomicrons, and secretion into lymph) (Lammert et al 2005; Wang et al 2007). Cholesterol appears to be specifically removed from the micelles as part of the absorption process: it is absorbed mainly in the duodenum and jejunum, but bile acids are not absorbed to an appreciable degree at these sites. Rather, specific bile acid transporters located in the ileum subsequently absorb bile acids, delivering them back to the liver and thus giving rise to an enterohepatic circulation (Levy et al 2007). Until recently non-cholesterol sterols were not thought to be absorbed during this process; 


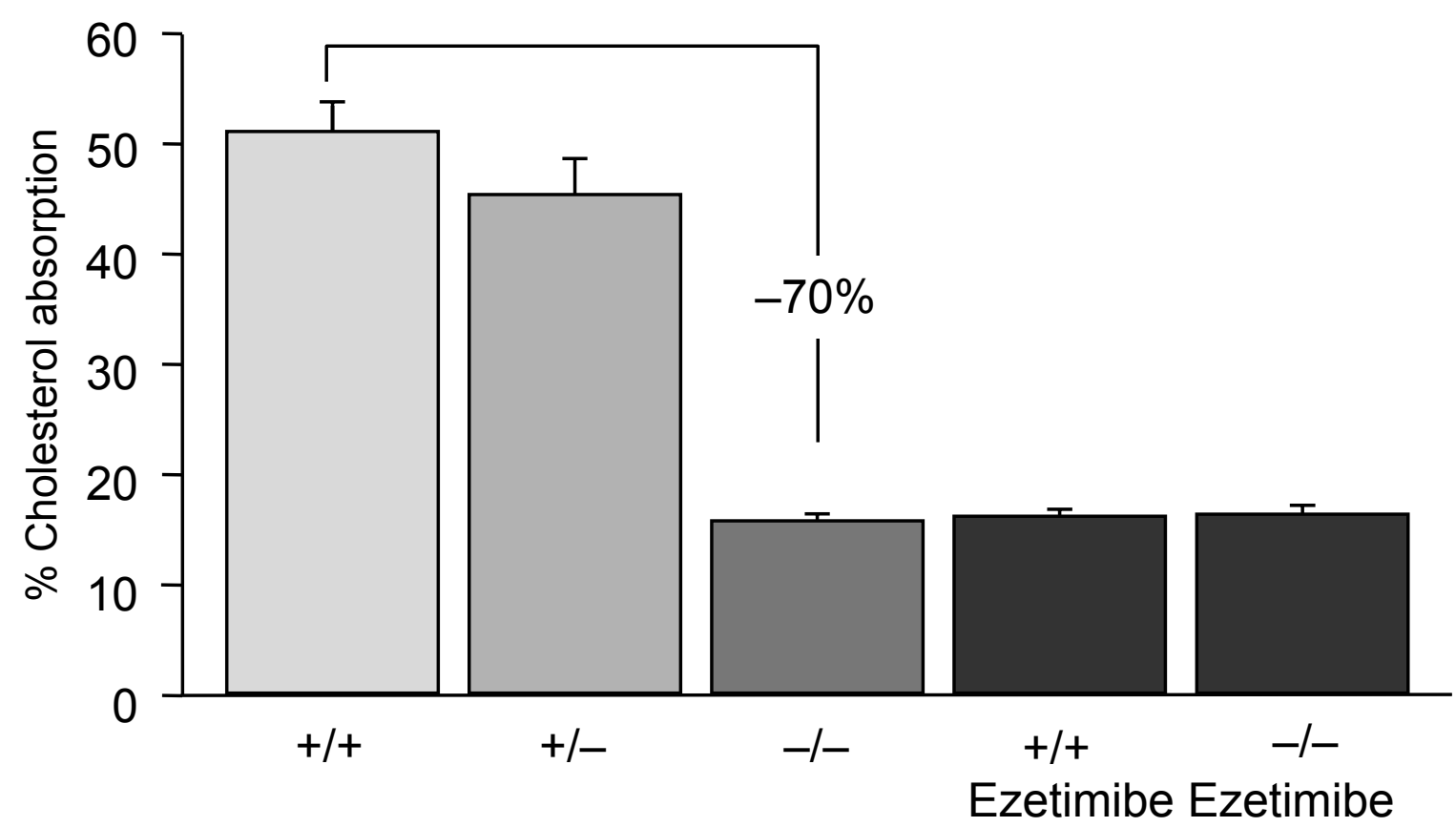

Figure I Cholesterol absorption in NPCILI (-/-) mice and in (+/+) mice treated with ezetimibe. Drawn from data of Altmann et al (2004); Garcia-Calvo et al (2005).

however, new insights into sitosterolemia or phytosterolemia, a rare autosomal recessive disorder caused by hyperabsorption and impaired biliary secretion of cholesterol and plant sterols, which subsequently accumulate in tissues causing tendon xanthoma and premature coronary artery disease, have depicted a different situation. The disease appears to be produced by mutations in two half-size $\mathrm{ABC}$ transporters, ABCG5 and ABCG8, localized at the apical membrane of the enterocyte and at the canalicular membrane in the liver. When functional, these transporters unite to form a full, active transporter and limit the absorption of both plant sterols and cholesterol by actively pumping them from enterocytes back into the intestinal lumen. These transporters may also promote the elimination of sterols in the liver by enhancing their excretion into bile. Mice lacking abcg5/abcg8 genes mimic the major phenotypes of human sitosterolemia (Salen et al 1992). A key protein involved in cholesterol absorption is NPC1L1, which has been found to be abundantly expressed in small intestine particularly in the jejunum at the brush-border membrane but also is present in subcellular compartments of human enterocyte, including lysosomes and mitochondria. NPC1L1 was first described in 2000 (Davies et al 2000); its name derives from the fact that it shares $42 \%$ amino acid identity with Niemann-Pick type C1 protein (NPC1), a protein involved in intracellular cholesterol transport and is also the causative gene for Niemann-Pick disease type $\mathrm{C} 1$ (Carstea et al 1997). In mouse, rat, and human, the small intestine showed a high level of NPC1L1 mRNA expression (Altmann et al 2004) (Figure 1). With the exception of human liver, which showed similar levels of expression as the intestine, NPC1L1 expression in all other tissues was $<10 \%$ of intestinal expression and was barely detectable in many tissues, in contrast with the fairly ubiquitous tissue expression of NPC1. Further analysis of the duodenal-ileal axis of rat small intestine demonstrated that peak expression of Npc1L1 mRNA and NPC1L1 protein occurred in the proximal jejunum, which was also the predominant site for sterol absorption (Altmann et al 2004).

The observation that mice lacking NPC1L1 have a markedly reduced sterol absorption confirmed the fundamental role of this protein as a cholesterol transporter in human enterocytes. After absorption, free cholesterol and fatty acids are re-esterified in the enterocyte by the action of acylcoenzyme A:cholesterol acyl-transferase (ACAT), packaged with triglycerides, phospholipids and apolipoprotein B-48 into chylomicrons, and finally secreted from the basolateral site of the enterocytes from where they enter the lymphatic channels and eventually are transported into the peripheral circulation (Wang et al 2007). Recent studies have significantly advanced our understanding of intestinal sterol absorption at the molecular level. Two nuclear hormone receptors are believed to be involved in the regulation of cholesterol homeostasis, the liver $\mathrm{X}$ receptor (LXR) and the farnesoid $X$ receptor (FXR). The natural ligands for LXR and FXR 
are oxysterols (oxidized derivatives of cholesterol) and bile acids, respectively (Russell et al 1999).

To modulate transcriptional activity, ligand-activated LXR or FXR form a heterodimer with one additional nuclear hormone receptor, the retinoid X receptor (RXR). These heterodimers control the transcription of several important genes that participate into cholesterol metabolism, sometimes appearing to antagonize the effects of each other. Two mechanism are involved in the reduction of cholesterol following FXR-RXR and LXR-RXR activation (Repa et al 2000, 2002). The FXR-RXR heterodimer suppresses CYP7A1 expression and decreases bile acid synthesis. Because non-polar lipids such as cholesterol have a limited solubility in the aqueous environment of the intestinal lumen, bile acids are required to solubilize these non-polar compounds and allow their absorption. By suppressing bile acid production, the activated FXR-RXR heterodimer decreases the solubilization and absorption of dietary cholesterol. Even though activation of the LXR-RXR heterodimer could not counterbalance the FXR-RXR-mediated suppression of CYP7A1 expression, the activated LXR-RXR heterodimer has a powerful effect on cholesterol homeostasis by inducing the expression of $\mathrm{ABC}$ transporters (specifically, $\mathrm{ABCA1}$ ) in enterocytes. This increase in ABCA1 expression represents the second mechanism by which the administration of the RXR ligand decreases cholesterol absorption. ABCA1 normally pumps cholesterol from enterocytes back out to the intestinal lumen, thereby limiting the amount of cholesterol absorbed (Repa et al 2000; Brewer and Santamarina-Fojo 2003). Indeed, mice treated with the RXR ligand present an increased intestinal expression of ABCA1, mediated by the activation of the LXR-RXR heterodimer. Given these findings, pharmacological activation of the nuclear hormone receptors RXR, LXR and FXR, including PPARs agonist that have been shown to positively affect LXR expression and activity, may represent a treatment option for hypercholesterolemia.

\section{Mechanism of action of ezetimibe}

In the past few years, an innovative approach to cholesterollowering therapy has been introduced. Ezetimibe is the first in a class of cholesterol-lowering agents with a mechanism of action that is very different from other lipid lowering therapies, including bile acid sequestrants.

By inhibiting cholesterol absorption at the level of the brush border of the intestine, ezetimibe reduces the amount of lipoprotein cholesterol circulated to the liver. In response to reduced cholesterol delivery, the liver reacts by upregulating
LDL-C receptors, which in turn leads to increased clearance of cholesterol from the blood (Lammert et al 2005).

A major advance in the understanding of ezetimibe's mechanism of action occurred in 2004 when Altmann and colleagues evaluated sequence data from human, rat, and mouse gastrointestinal cDNA libraries to identify proteins with features, such as transmembrane domains and known cholesterol sensing motifs, that would be expected to be seen in a putative cholesterol transporter (Altmann et al 2004).

Interestingly, ezetimibe has also been shown to block the uptake of oxidized LDL by human macrophages through inhibition of both NPC1L1 and ANX2/CAV1 (Seedorf et al 2004; Levy et al 2007), suggesting the actions of this agent may extend beyond the intestinal epithelium. Aminopeptidase $\mathrm{N}$ (CD13) in the brush border membrane may represent an additional molecular target for ezetimibe. Ezetimibe has been shown to bind to aminopeptidase $\mathrm{N}$ thereby blocking the endocytosis of cholesterol from micelles in the intestinal lumen (Levy et al 2007). Additional evidence has come from in vitro binding assays that tested directly the interaction between the drug and NPC1L1. Garcia-Calvo, with Davis, Thornbury, and colleagues (Garcia-Calvo et al 2005) developed a binding assay and showed that labeled ezetimibe glucuronide bound specifically to a single site in intestinal epithelial brush border membranes and in embryonic kidney cells engineered to express NPC1L1. Furthermore, the binding affinities of ezetimibe and its analogs to recombinant NPC1L1 were indistinguishable from those observed for native enterocyte membranes. Values for the dissociation constant of ezetimibe glucuronide for NPC1L1 were evaluated in intestinal enterocyte membranes and human embryonic kidney cells engineered to express NPC1L1 from various species. Binding affinities were highest for rhesus monkey compared with more moderate values for rat and human, with the lowest values reported for mouse, which also correlates well with the in vivo potency observed for ezetimibe across species. Also, ezetimibe failed to affect cholesterol absorbtion in $\mathrm{NpClL1}^{-1_{-}}$null mice compared with wild-type mice (Figure 2). These results established NPC1L1 as the target for the action of ezetimibe.

Ezetimibe is rapidly metabolized in the intestine to its phenolic glucuronide; once glucuronidated, it is excreted in the bile, thereby delivering the drug back to the main site of action. Cholesterol absorption studies indicated that the glucuronide appeared more potent than ezetimibe itself, and this is likely because glucuronidated ezetimibe localizes more avidly to the intestine. Autoradiographic analysis demonstrated that drugrelated material was located throughout the intestinal villi but 


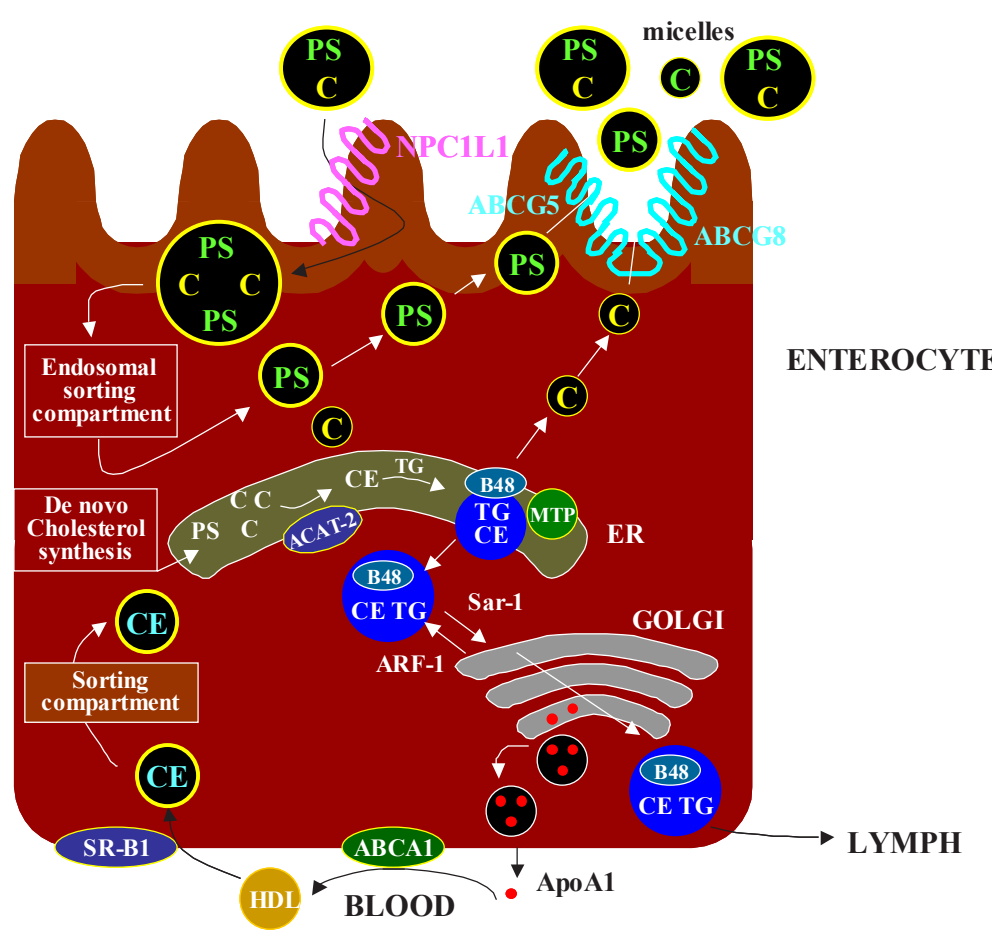

Figure 2 Overview of the principal steps in the intestinal absorption of cholesterol (C). The intraluminal phase involves the digestion/hydrolysis of dietary lipids and micellar solubilization of cholesterol. The membrane transport phase involves cholesterol release from micelles at the brush border membrane and uptake into enterocytes via several sterol transporters, including Niemann-Pick CIlike I protein (NPCILI), aminopeptidase N (CDI3), and annexin-2/caveolin-I (ANX2/CAVI). The brush border membrane also contains ATP-binding cassette (ABC) transporters (ABCG5 and ABCG8), which primarily move plant sterols and to a lesser extent cholesterol out of the enterocytes.

concentrated in the villus tip. In humans, ezetimibe is rapidly absorbed and primarily metabolized in the small intestine and liver to its glucuronide, with little oxidative cytocrome P450 mediated metabolism (Patrick et al 2002). Ezetimibe and its glucuronide undergo enterohepatic recycling and have a halflife of approximately 24 hours in humans. Ezetimibe and/or the glucuronide metabolite are excreted in the feces $(90 \%)$ and urine (10\%). Since ezetimibe does not influence the activities of cytocrome $\mathrm{P} 450$ enzymes, significant pharmacokinetic interactions with many medications have not been noted. Pharmacokinetic interaction studies of ezetimibe in humans have found no significant changes in the plasma levels of other medications including statins (atorvastatin, simvastatin, pravastatin, lovastatin, and fluvastatin), fibrates (gemfibrozil and fenofibrate), digoxin, glipizide, warfarin and oral contraceptives (ethinyl estradiol and levonorgestrel (Kosoglou et al 2005).

\section{Treating two sources of cholesterol: co-administering ezetimibe together with a statin}

Statins (eg, fluvastatin, lovastatin, pravastatin, simvastatin, atorvastatin, and rosuvastatin) deliver only single inhibition of cholesterol production in the liver by blocking HMG-Co A reductase, the rate-limiting step in cholesterol synthesis, but do not impact significantly intestinal cholesterol absorption (Shepherd et al 2004). As a result, hepatocytes become depleted of cholesterol and respond by increasing LDL-C clearance from the blood (via upregulation of hepatic LDL-C receptors) and decreasing entry of LDL-C into the circulation. These actions, in turn, result in the lowering of plasma LDL-C levels. Of note HMGCoA reductase inhibitors could target also cholesterol synthesis in the ileum (Parker et al 1990; Sviridov et al 1990); therefore reduced cholesterol biosynthesis in the small intestine by statins may also contribute to the reduction of plasma cholesterol concentrations.

The use of two lipid-lowering compounds (ezetimibe co-administered with a statin) with complementary mechanisms of action provides a powerful new approach to prevent and treat atherosclerosis. Treating two sources of cholesterol (rather than one source with statins or ezetimibe alone) results in a substantially greater overall LDL-C lowering efficacy as well as in more patients attaining or getting below LDL-C goals.

Ezetimibe added to a statin has the potential to lower cholesterol by dual inhibition of both cholesterol absorption 
and cholesterol synthesis. Adding ezetimibe to ongoing statin therapy led to a substantial additional reduction in LDL cholesterol levels, facilitating attainment of NCEP goals. In patients with hypercholesterolemia not at goal on statin therapy alone, the Ezetimibe Add-On to Statin for Effectiveness (EASE) trial (Pearson et al 2004; Denke et al 2006) demonstrated that co-administering ezetimibe (10 mg) with any dose of statin reduced LDL-C levels by an additional $25 \%$, compared with the usual $6 \%$ attained by doubling the statin dose and improved LDL-C goal attainment from $20 \%$ on statin monotherapy to $71 \%$ vs $18.9 \%$ on statin alone, $\mathrm{p}<0.001$ (Gagne et al 2002) (Figures 3 and 4).

The benefits were consistent across the statin brand and dose subgroups, in particular association of ezetimibe with atorvastatin (10-80 mg), fluvastatin (20-80 mg), lovastatin (20-80 mg), pravastatin (10-80 mg), simvastatin (10-80 mg) was studied (Denke et al 2006).

Ezetimibe when co-administered with a statin was significantly better than placebo in increasing high-density lipoprotein cholesterol (HDL-C) and reducing triglycerides, non-HDL-C, and apolipoprotein B ( $p<0.001$ for all between-treatment differences). The superior reduction in LDL-C with ezetimibe compared with placebo was consistent across all ages, in men and women, and all ethnicities. Patients with diabetes or metabolic syndrome (including those with or without diabetes) who were randomized to ezetimibe had superior reduction in LDL-C compared with placebo. The superior LDL-C reduction with ezetimibe was consistent when analyzed by statin brand and statin dose.

Other lipid parameters statistically improved with the addition of ezetimibe were: ratios of LDL-C/HDL-C, of total cholesterol/HDL-C and non-HDL-C. Finally C-reactive protein, a non-specific inflammatory marker associated with cardiovascular risk, was further reduced by $12 \%$.

Many studies have centered on the use of ezetimibe/ simvastatin as a single tablet: the association was superior to atorvastatin alone in reducing plasma LDL-C at every dose comparison studied, according to double-blind, randomized, forced-titration study (Ballantyne et al 2004). Adult patients with hypercholesterolemia experienced significantly greater reductions in LDL-C when they received the typical starting dose of ezetimibe/simvastatin 10/20 mg (50\%) compared with atorvastatin $10 \mathrm{mg}(37 \%)$ after 6 weeks of treatment ( $p<0.001)$. Interestingly, the reduction in LDL-C levels seen with the association was comparable to the $50 \%$ reduction in LDL-C levels that has shown to be required for reversal of IVUS-evidenced atherosclerosis (Pearson et al 2004). In addition, ezetimibe/ simvastatin resulted in significantly more patients achieving LDL-C goal $(<100 \mathrm{mg} / \mathrm{dL})$ whether compared with atorvastatin or simvastatin alone (Feldman et al 2004; Ballantyne et al 2005) (Figure 5).

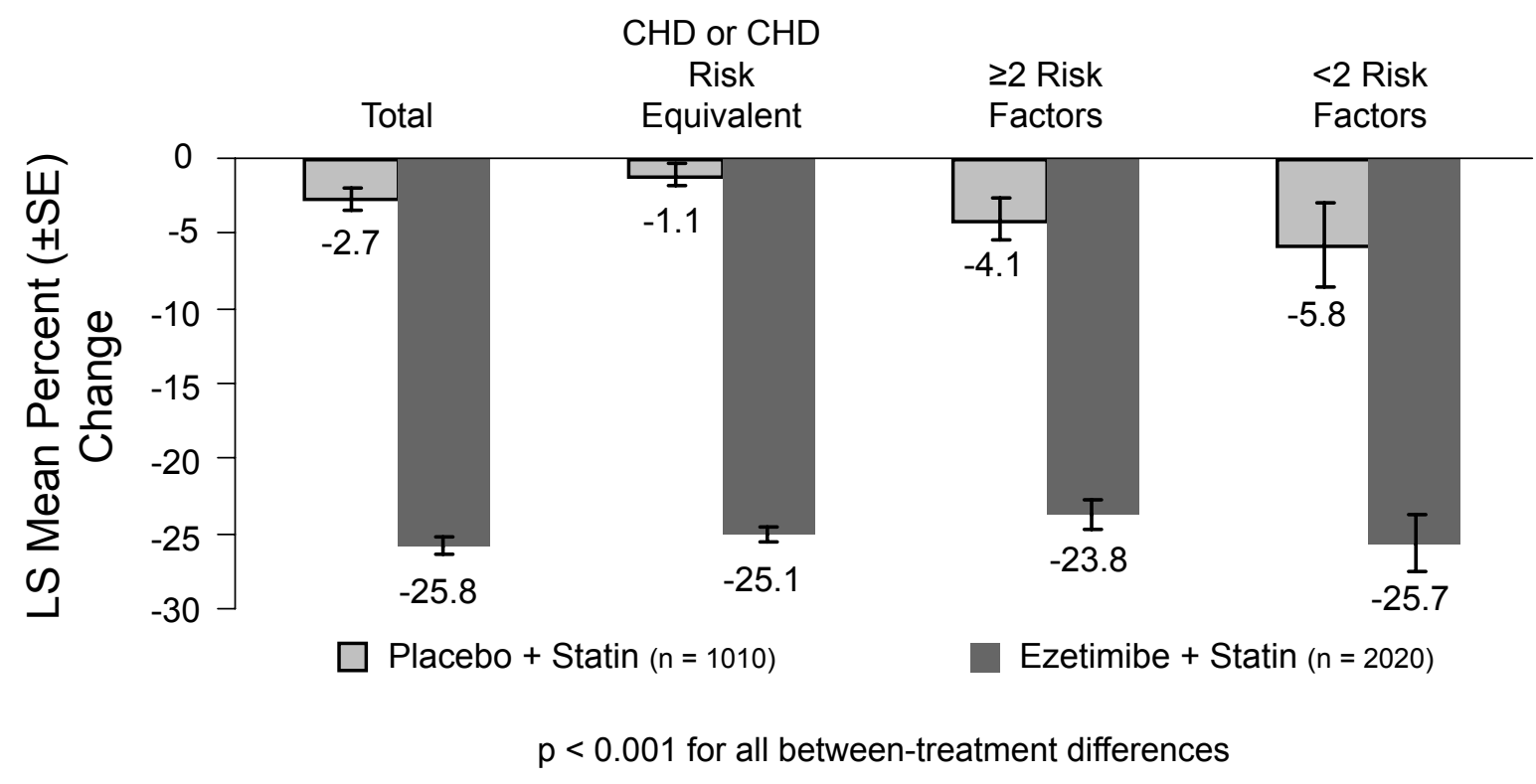

Figure 3 Ezetimibe Add-On to Statin for Effectiveness study: percentage changes in LDL-C overall and by NCEP CHD risk category $(\mathrm{n}=3030)$. Drawn from data of Pearson et al (2005). 


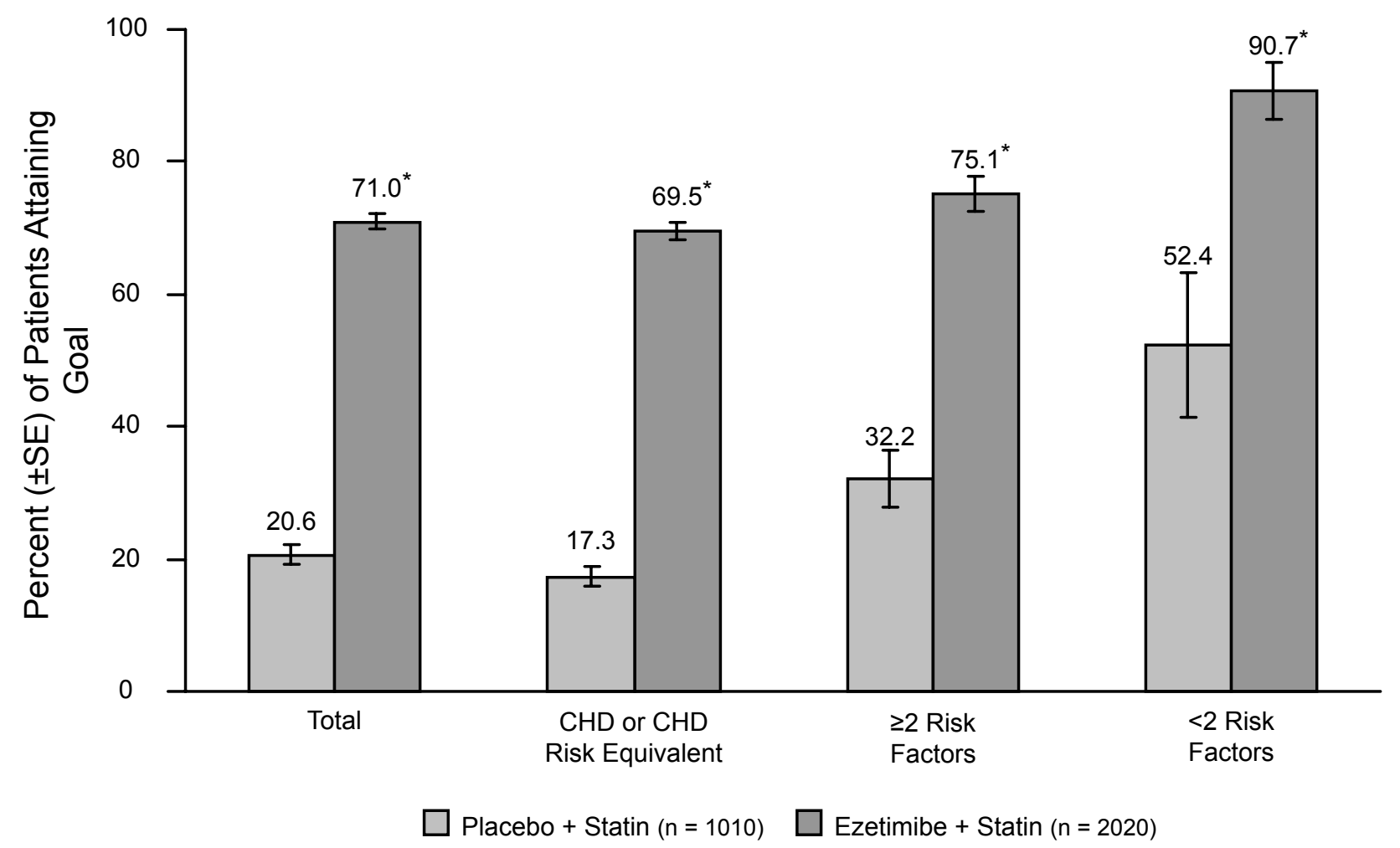

$$
\mathrm{p} \leq 0.002 \text { placebo }+ \text { statin vs ezetimibe }+ \text { statin }
$$

Figure 4 Ezetimibe Add-On to Statin for Effectiveness study: LDL-C goal attainment for patients $(n=3030)$ not at goal at baseline. Drawn from data of Pearson et al (2005).

\section{Statin dose}

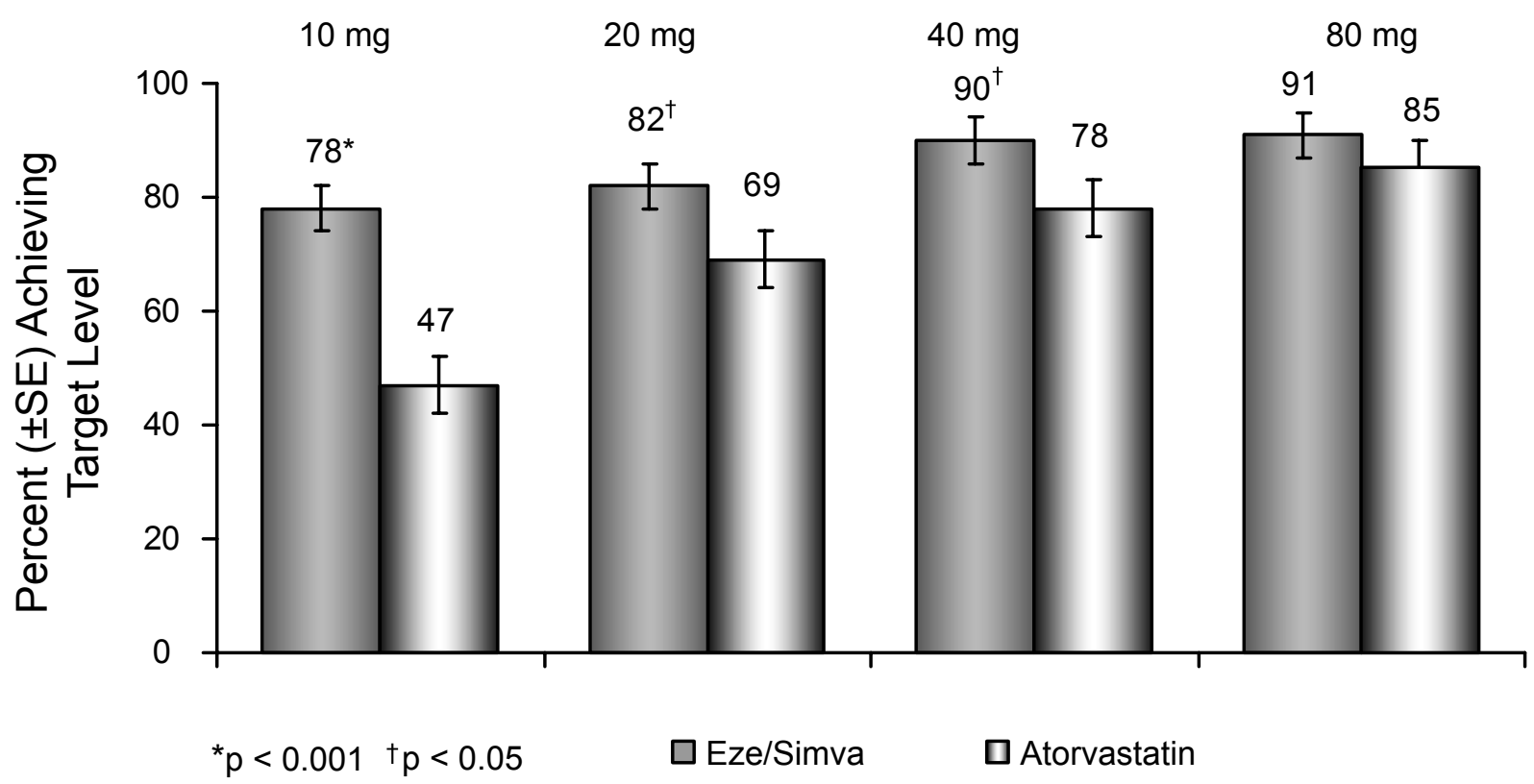

Figure $\mathbf{5}$ Vytorin Versus Atorvastatin Study:Achievement of LDL-C $<100 \mathrm{mg} / \mathrm{dL}$ in patients with CHD risk equivalent. Drawn from data of Ballantyne et al (2005). 
Further, compared with patients receiving atorvastatin alone, more patients treated with ezetimibe/simvastatin reached the more aggressive LDL-C target $(<70 \mathrm{mg} / \mathrm{dL}$ ), as recently recommended by the NCEP for very high-risk CHD patients (Ballantyne et al 2005) (Figure 6). Approximately $23 \%$ of patients receiving atorvastatin $40 \mathrm{mg}$ alone and $36 \%$ of patients receiving atorvastatin $80 \mathrm{mg}$ alone reached the optional LDL-C treatment target of $<70 \mathrm{mg} / \mathrm{dL}$ compared with $57 \%$ of patients treated with ezetimibe/simvastatin (10/40 $\mathrm{mg}$ ) and $64 \%$ of patients receiving ezetimibe/simvastatin (10/80 mg) (Ballantyne et al 2005).

The treatment with the usual starting doses of ezetimibe/ simvastatin $10 / 20 \mathrm{mg}$ resulted in a $51 \%$ reduction of LDL-C compared to $36 \%$ reduction of LDL-C with the starting dose of atorvastatin $10 \mathrm{mg}$ (Figure 7).

Similar data were observed in patients treated with ezetimibe/simvastatin compared to rosuvastatin (Catapano et al 2006); patients receiving ezetimibe/simvastatin had statistically significantly greater reductions in lowering LDL-C at all doses and across doses (52\%-61\%) compared with a potent statin monotherapy agent, rosuvastatin, $(46 \%-57 \%$; $\mathrm{p}<0.001)$ at the usual starting, next highest, and maximum doses (Figure 8).

Significantly higher percentages of patients achieved LDL-C levels of $<100 \mathrm{mg} / \mathrm{dL}$ ( $2.6 \mathrm{mmol} / \mathrm{L})$ with ezetimibe/ simvastatin versus rosuvastatin.

Furthermore, the greater LDL-C reducing efficacy produced by ezetimibe/simvastatin generally resulted in a higher percent of LDL-C goal attainment, particularly for those high-risk patients who attained LDL-C levels $<70 \mathrm{mg} /$ $\mathrm{dL}(<1.8 \mathrm{mmol} / \mathrm{L})$. Patients treated with ezetimibe/simvastatin versus rosuvastatin showed significantly greater improvements in TC, apo B, non-HDL-C, and LDL-C:HDL-C and TC:HDL-C ratios at all dose comparisons, and also in TG at the usual starting doses (10/20 mg versus $10 \mathrm{mg})$, the maximum doses (10/80 $\mathrm{mg}$ versus $40 \mathrm{mg}$ ), and when averaged across all doses. Improvements in HDL-C and hsCRP were similar for both study medications at all dose comparisons. The EXPLORER study evaluated whether the association of rosuvastatin $40 \mathrm{mg}$ with ezetimibe $10 \mathrm{mg}$ enables more high-risk patients to achieve the LDL target compared with rosuvastatin monotherapy. This study demonstrated that the addition of ezetimibe increased goal achievement to $94 \%$ with 70\% mean reduction in LDL-C (Ballantyne et al 2006). Similar results were obtained in another small study, in severe hyperlipidemic patients, resistant to $40 \mathrm{mg}$ rosuvastatin monotherapy. LDL-C levels were reduced by $51 \%$ with the association rosuvastatin $40 \mathrm{mg}$ /ezetimibe $10 \mathrm{mg}, 45 \%$ of the patients achieved LDL-C values below $100 \mathrm{mg} / \mathrm{dL}$ (Leibovitz et al 2006). The VYTAL study compared the efficacy and safety of the recommended usual starting and next highest doses of ezetimibe/simvastatin and atorvastatin in patients with diabetes mellitus and hypercholesterolemia (Goldberg et al 2006). The association of ezetimibe/simvastatin provided significantly greater mean reduction in LDL-cholesterol levels $10 / 20 \mathrm{mg}(-54 \%)$ vs atorvastatin $10 \mathrm{mg}(-38 \%$, p < 0.001)

\section{Statin dose}

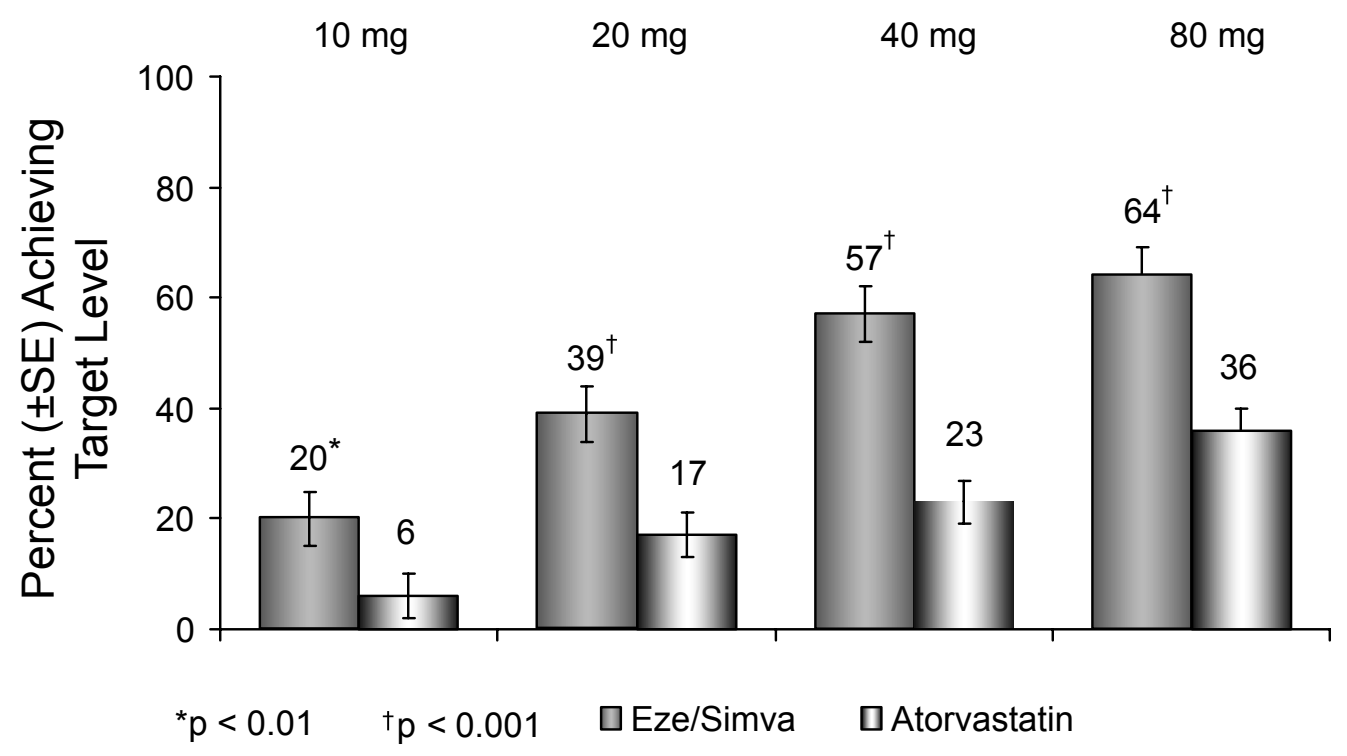

Figure 6 Vytorin Versus Atorvastatin Study:Achievement of LDL-C $<70 \mathrm{mg} / \mathrm{dL}$ in patients with CHD risk equivalent. Drawn from data of Ballantyne et al (2005). 


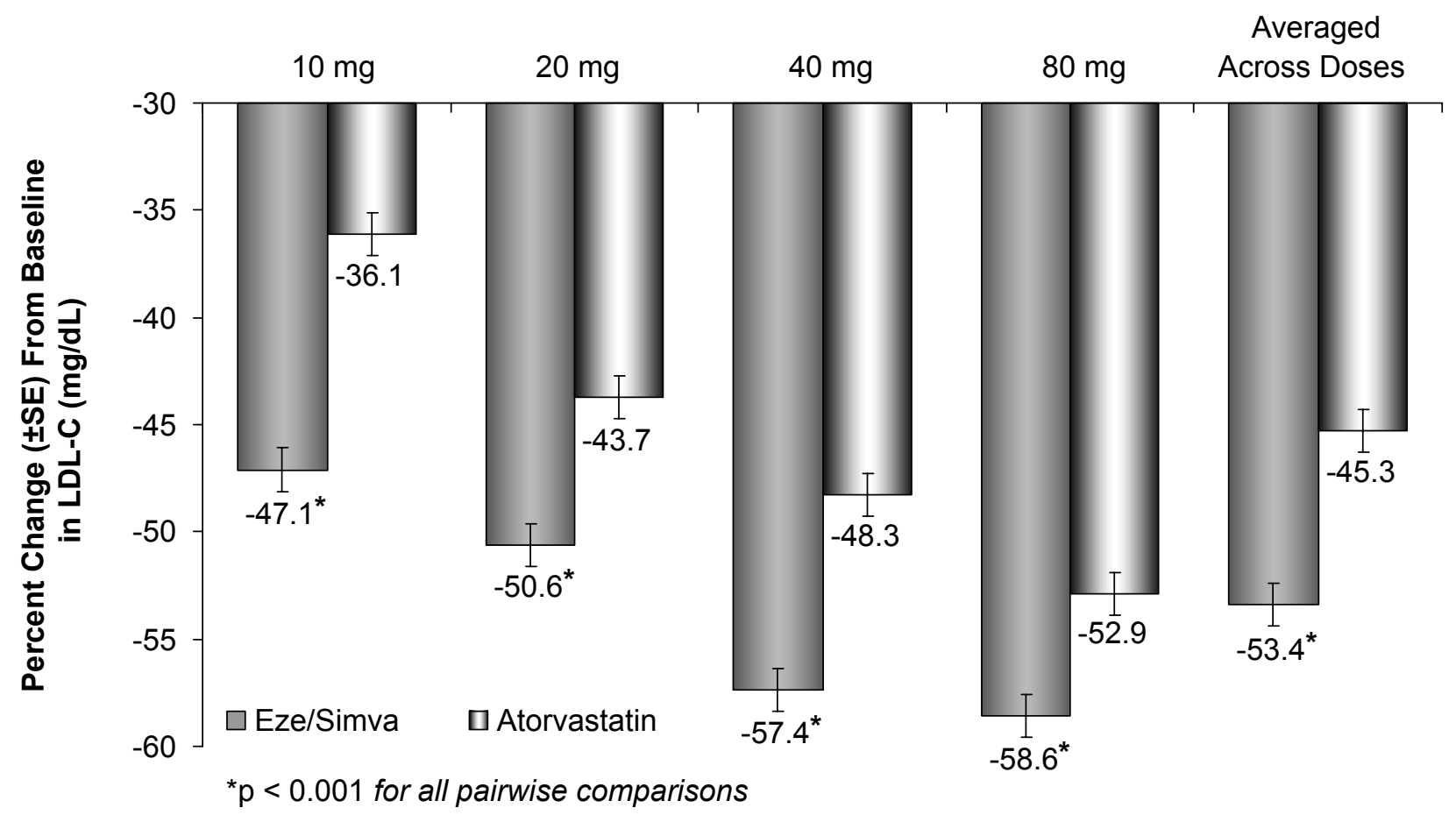

Treatment comparison at each dose and averaged across doses

Figure 7 Vytorin Versus Atorvastatin Study: LDL-C reductions. Drawn from data of Ballantyne et al (2005).

or $20 \mathrm{mg}(-45 \%, \mathrm{p}<0.001)$ and with ezetimibe $10 / 40 \mathrm{mg}$ $(-58 \%)$ vs atorvastatin $40 \mathrm{mg}(-51 \%, \mathrm{p}<0.001)$. Ezetimibe/ simvastatin was also superior to atorvastatin in attainment of LDL-cholesterol levels less than $70 \mathrm{mg} / \mathrm{dL}(\mathrm{p}<0.001$ for all doses comparisons).
When administered in monotherapy, ezetimibe $10 \mathrm{mg} / \mathrm{die}$ showed a similar adverse events (AE) profiles compared to placebo (Dujovne et al 2002). Most of the adverse events (AEs) were judged by the investigators to be mild or moderate at their greatest intensity. Overall a similar percentage of patients

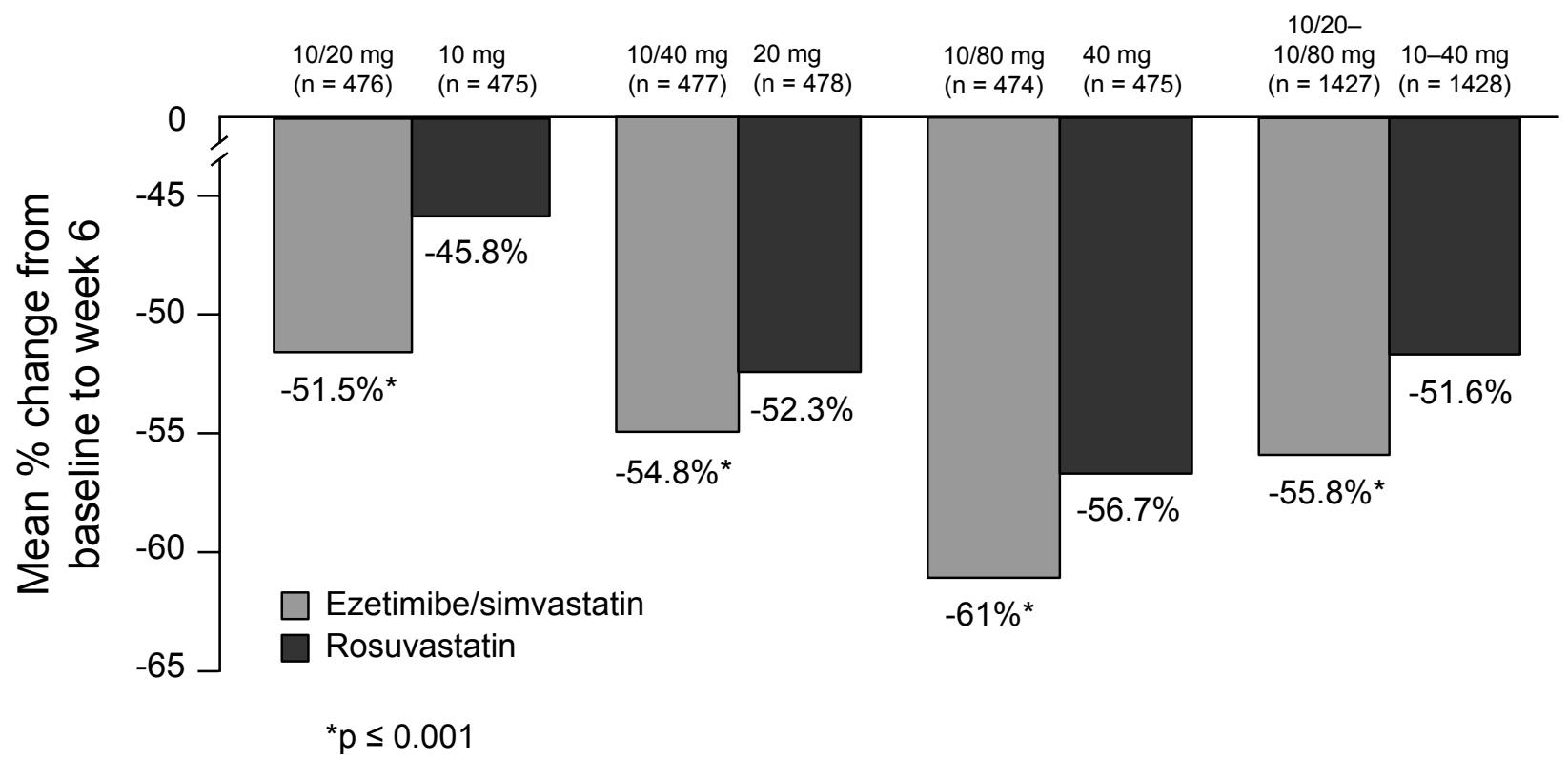

Figure 8 LDL-C reduction across the dose range. Drawn from data of Catapano et al (2006). 
reported AEs in ezetimibe group then in the placebo group (63\% vs 66\%). The nature, number and pattern of occurencies of AEs that led to study discontinuation suggested no differential risk with ezetimibe treatment related to placebo; $3 \%$ of placebo and $4 \%$ of ezetimibe recipients discontinued due to AEs. Laboratory test results were generally similar between treatment groups. The incidence of elevated laboratory function test values (ALT and/or AST $>3 x$ the upper limit of normal (ULN) at two consecutive visits $>1$ week apart) was $<1 \%$ in both placebo and ezetimibe treatment groups. Elevated muscle enzyme values $(\mathrm{CK}>10 \mathrm{x}$ ULN) were reported in less than $1 \%$ of groups. All the observed increases were asymptomatic and either transient with continued treatment or reversible following treatment discontinuation.

When associated with statins, approximately 95\% of patients completed the treatment (Gagne et al 2002). Gastrointestinal system disorder was the most common AE leading to discontinuation, occurring in $1 \%$ of patients. In phase II and III studies of the statin plus ezetimibe group, $20 \%$ of patients had what the investigator considered to be treatment-related $\mathrm{AE}$ compared to $17 \%$ in the group of patients receiving statin and placebo $(10 \%$ of AE incidence was related to gastrointestinal system disorders). Elevation in either ALT or AST $>3 x$ ULN at two consecutive posline measurements were similar in the two treatment groups ( $1 \%$ and $<1 \%$ respectively). No case of rhabdomyolysis was reported in ether treatment group.

In the EASE study ezetimibe add-on to statin was well tolerated with a safety profile comparable with placebo (Denke et al 2006). When adverse events were evaluated for each of statin groups (simvastatin, atorvastatin, and other), no particular pattern was found among patients receiving ezetimibe versus placebo for any adverse event category (Gagne et al 2002). Results of additional measurements of safety revealed no evidence of any adverse effects of ezetimibe treatment compared with placebo relative to statin monotherapy. In general, there were no significant differences between ezetimibe/simvastatin and rosuvastatin (Catapano et al 2006) in terms of clinically significant elevations in levels of muscle or liver enzymes. With regard to the liver enzymes ALT and AST, ezetimibe/simvastatin tended towards a numerically higher frequency of elevated levels, compared with rosuvastatin, partially attributed to differences at the 10/80 mg and $40 \mathrm{mg}$ doses, but not at lower doses. Both study drugs were generally well tolerated. In conclusion, while the combination of ezetimibe with moderate doses of statins appears to be reasonably safe, the long-term safety of combination with high doses of statins remains to be established (Davidson et al 2007). More data are expected from the ongoing trials described below.

\section{Ezetimibe, dyslipidemia and cardiovascular outcomes: future developments}

Although treating two sources of cholesterol through dual inhibition provides superior LDL-C lowering efficacy with improved LDL-C goal attainment, one key clinical question remains to be addressed: Do the lower LDL-C levels achieved with dual inhibition of cholesterol production and absorption ultimately translate into reduced cardiovascular or renal events and a slower rate of progression of atherosclerosis? This question is currently being assessed in several major cardiovascular outcomes studies, including Ezetimibe and Simvastatin in Hypercholesterolemia Enhances Atherosclerosis Regression (ENHANCE), Improved Reduction of Outcomes: Vytorin ${ }^{\mathrm{TM}}$ Efficacy International Trial (IMPROVE IT), Simvastatin and Ezetimibe in Aortic Stenosis Study (SEAS), and Study of Heart and Renal Protection (SHARP). These major endpoint trials collectively involve more than 21,000 patients.

More recently ezetimibe has been co-administered with fenofibrate in patients with mixed hyperlipidemia (McKenney et al 2006). Preliminary results after completing the 12-week randomized double-blind base study showed that the association of ezetimibe to fenofibrate produced significantly greater reduction in LDL-C $(-22 \%$ vs $-9 \%$ respectively; $\mathrm{p}<0.001)$. There were also significantly improvements in TG, HDL, total cholesterol and non-HDL-C with fenofibrate + ezetimibe compared with fenofibrate alone. Overall fenofibrate plus ezetimibe was well tolerated and the proportion of patients with consecutive elevations of ALT or AST $>3 x$ the normal limit was similar (1.2\% fenofibrate + ezetmibe vs $1.7 \%$ fenobibrate alone). The efficacy of the association of ezetimibe/simvastatin 10/20 mg with fenofibrate $160 \mathrm{mg}$ has been investigated in patients with mixed hyperlipidemia (Farnier et al 2007). LDL-C was significantly reduced with eze/simva + feno $(-45.8 \%)$ compared with feno $(-15.7 \%)$ or placebo $(-3.5 \%)$. Treatment with eze/simva + feno was generally well tolerated with a safety profile similar to the eze/simva and feno therapies. Further studies are warranted to investigate the role of ezetimibe and fibrates in mixed dyslipidemia.

\section{Conclusions}

A wide therapeutic gap exists between target LDL-C levels and LDL-C levels typically achieved in actual clinical practice - a 
gap that will certainly widen with traditional therapy of single inhibition in light of recent amendments to the NCEP ATP III guidelines. The new aggressive cholesterol treatment goals call for a more advanced therapeutic approach to maximize the cardiovascular benefits associated with lower LDL-C levels. One logical approach is to target both cholesterol production in the liver and absorption in the intestine. By administrating ezetimibe/simvastatin as a single tablet or co-administering ezetimibe together with any dose of any statin, we can expect superior LDL-C-lowering efficacy and a substantially greater proportion of patients achieving or getting below LDL-C treatment goals. Treating two sources of cholesterol through dual inhibition should therefore be considered as a more advanced therapeutic option for all hypercholesterolemic patients whose LDL-C levels are not appropriately controlled approximately 2-3 months after initiating statin monotherapy.

\section{References}

Altmann SW, Davis HR Jr, Zhu LJ, et al. 2004. Niemann-Pick C1 Like 1 protein is critical for intestinal cholesterol absorption. Science, 303:1201-4.

[Anon] 1984. The Lipid Research Clinics Coronary Primary Prevention Trial results. I. Reduction in incidence of coronary heart disease. JAMA, 251:351-64.

[Anon] 1994. Randomised trial of cholesterol lowering in 4444 patients with coronary heart disease: the Scandinavian Simvastatin Survival Study (4S). Lancet, 344:1383-9.

Ballantyne C, Abate N, Zhong Y, et al. 2005. Dose-comparison study of the combination of ezetimibe and simvastatin (Vytorin) versus atorvastatin in patients with hypercholesterolemia. the Vytorin Versus Atorvastatin (VYVA Study). Am Heart J, 149:464-73.

Ballantyne C, Sosef F, Duffield E. 2006. Efficacy and safety of rosuvastatin plus ezetimibe in high-risk patients: results from the EXPLORER study [abstract]. Atherosclerosis Supplement, 7:552.

Ballantyne CM, Blazing MA, King TR, et al. 2004. Efficacy and safety of ezetimibe co-administered with simvastatin compared with atorvastatin in adults with hypercholesterolemia. Am J Cardiol, 93:1487-94.

Ballantyne CM, Houri J, Notarbartolo A, et al. 2003. Effect of ezetimibe coadministered with atorvastatin in 628 patients with primary hypercholesterolemia: a prospective, randomized, double-blind trial. Circulation, 107:2409-15.

Brewer HB Jr, Santamarina-Fojo S. 2003. New insights into the role of the adenosine triphosphate-binding cassette transporters in high-density lipoprotein metabolism and reverse cholesterol transport. Am J Cardiol, 91:3E-11E.

Carstea ED, Morris JA, Coleman KG, et al. 1997. Niemann-Pick C1 disease gene: homology to mediators of cholesterol homeostasis. Science, 277:228-31.

Castelli WP, Anderson K, Wilson PW, et al. 1992. Lipids and risk of coronary heart disease. The Framingham Study. Ann Epidemiol, 2:23-8.

Catapano AL, Davidson MH, Ballantyne CM, et al. 2006. Lipid-altering efficacy on the ezetimibe/simvastatin single tablet versus rosuvastatin in hypercholesterolemic patients. Curr Med Res Opin, 22:2041-53.

Collins R, Armitage J, Parish S, et al. 2004. Effects of cholesterol-lowering with simvastatin on stroke and other major vascular events in 20536 people with cerebrovascular disease or other high-risk conditions. Lancet, 363:757-67.

Cook RP. 1958. Cholesterol. Distribution of sterols in organisms and in tissues. In Cook RP, ed. Cholesterol. Chemistry, biochemistry, and pathology. New York: Academic Press. p. 145-80.
Davidson MH, Robinson JG. 2007. Safety of aggressive lipid management. $J$ Am Coll Cardiol, 49:1753-62.

Davies JP, Ioannou YA. 2000. Topological analysis of Niemann-Pick C1 protein reveals that the membrane orientation of the putative sterolsensing domain is identical to those of 3-hydroxy-3-methylglutaryl-CoA reductase and sterol regulatory element binding protein cleavageactivating protein. $J$ Biol Chem, 275:24367-74.

Denke M, Pearson T, McBride P, et al. 2006. Ezetimibe added to ongoing statin therapy improves LDL-C goal attainment and lipid profile in patients with diabetes or metabolic syndrome. Diabetes Vasc Dis Res, 3:93-102.

Dietschy JM, Turley SD, Spady DK. 1993. Role of liver in the maintenance of cholesterol and low density lipoprotein homeostasis in different animal species, including humans. J Lipid Res, 34:1637-59.

Downs JR, Clearfield M, Tyroler HA, et al. 2001. Air Force/Texas Coronary Atherosclerosis Prevention Study (AFCAPS/TEXCAPS): additional perspectives on tolerability of long-term treatment with lovastatin. $\mathrm{Am}$ J Cardiol, 87:1074-9.

Dujovne CA, Ettinger MP, McNeer JF, et al. 2002. Efficacy and safety of potent new selective cholesterol absorbtion inhibitor, ezetimibe in patients with primary hypercholesterolemia. Am J Cardiol, 90:1092-7.

Farnier M, Roth E, Gil-Extremera B, et al. 2007. Ezetimibe/Simvastatin + Fenofibrate Study Group..Efficacy and safety of the coadministration of ezetimibe/simvastatin with fenofibrate in patients with mixed hyperlipidemia. Am Heart J, 153:335.e1-8.

Feldman T, Koren M, Insull W Jr, et al. 2004. Treatment of high-risk patients with ezetimibe plus simvastatin co-administration versus simvastatin alone to attain National Cholesterol Education Program Adult Treatment Panel III low-density lipoprotein cholesterol goals. Am J Cardiol, 93:1481-6.

Foley KA, Simpson RJ Jr, Crouse JR III, et al. 2003. Effectiveness of statin titration on low-density lipoprotein cholesterol goal attainment in patients at high risk of atherogenic events. Am J Cardiol, 92:79-81.

Frick MH, Elo O, Haapa K, et al. 1987. Helsinki Heart Study: primaryprevention trial with gemfibrozil in middle-aged men with dyslipidemia. Safety of treatment, changes in risk factors, and incidence of coronary heart disease. N Engl J Med, 317:1237-45.

Gagne C, Bays HE, Weiss SR, et al. 2002. Efficacy and safety of ezetimibe added to ongoing statin therapy for treatment of patients with primary hypercholesterolemia. Am J Cardiol, 90:1084-91.

Gagne C, Bays HE, Weiss SR, et al. 2002. Efficacy and safety of ezetimibe added to ongoing statin therapy for treatment of patients with primary hypercholesterolemia. Am J Cardiol, 90:1084-91.

Garcia-Calvo M et al. 2005. The target of ezetimibe is Niemann-Pick C1Like 1 (NPC1L1). Proc Natl Acad Sci USA, 102:8132-7.

Goettsch WG, Yin DD, Alemao E, et al. 2004. Statins are less effective in common daily practice among patients with hypercholesterolemia: the REALITY-PHARMO study. Curr Med Res Opin, 20:1025-33.

Goldberg RB, Guyton JR, Mazzone T, et al. 2006. Ezetimibe/simvastatin vs atorvastatin in patients with type 2 diabetes mellitus and hypercholesterolemia: the Vytal study. Mayo Clin Proc, 81:1579-88.

Goldstein JL, Brown MS. 1990. Regulation of mevalonate pathway. Nature, 343:425-30.

Grundy SM, Cleeman JI, Merz CN, et al. 2004. Implications of recent clinical trials for the National Cholesterol Education Program Adult Treatment Panel III Guidelines. J Am Coll Cardiol, 44:720-32.

Illingworth DR, Duell PB, Connor WE. 1995. Disorders of lipid metabolism. In Felig P, Baxter JD, Frohman LA, eds. Endocrinology and metabolism. New York: McGraw-Hill. p. 1315-403.

Kerzner B, Corbelli J, Sharp S, et al. 2003. Efficacy and safety of ezetimibe coadministered with lovastatin in primary hypercholesterolemia. Am J Cardiol, 91:418-24.

Kosoglou T, Statkevich P, Johnson-Levonas AO, et al. 2005. Ezetimibe: a review of its metabolism, pharmacokinetics and drug interactions. Clin Pharmacokinet, 44:467-94. 
Lammert F, Wang DQH. 2005. New insights into the genetic regulation of intestinal cholesterol absorbtion. Gastroenterology, 129:718-34.

Lavoie MA. 2003. Ezetimibe and cholesterol absorption. Can J Clin Pharmacol, 10(Suppl A):7A-12A.

Leibovitz E, Gavish D, Bitzur R, et al. 2006. Efficacy and safety of rosuvastatin-ezetimibe combination for the treatment of severe hypercholesterolemia [abstract]. Atheroscler Suppl, 7:554 (abstract).

Levy E, Spahis S, Sinnet D, et al. 2007. Intestinal cholesterol transport proteins: an update and beyond. Curr Opin Lipidol, 18:310-18

Manninen V, Elo MO, Frick MH, et al. 1988. Lipid alterations and decline in the incidence of coronary heart disease in the Helsinki Heart Study. JAMA, 260:641-51.

McKenney JM, Farnier M, Lo KW, et al. 2006. Safety and efficacy of longterm co-administration of fenofibrate and ezetimibe in patients with mixed hyperlipidemia. J Am Coll Cardiol, 47:1584-7.

Melani L, Mills R, Hassman D, et al. 2003. Efficacy and safety of ezetimibe coadministered with pravastatin in patients with primary hypercholesterolemia: a prospective, randomized, double-blind trial. Eur Heart $J$, 24:717-28.

Neal RC, Jones PH. 2003. Lipid-lowering: can ezetimibe help close the treatment gap? Cleve Clin J Med, 70:777-83.

Parker RA, Lark RW, Sit SY, et al. 1990. Selective inhibition of cholesterol synthesis in liver versus extrahepatic tissues by HMG-CoA reductase inhibitors. J Lipid Res, 31:1271-82.

Patrick JE, Kosoglou T, Stauber KL, et al. 2002. Disposition of the selective cholesterol absorption inhibitor ezetimibe in healthy male subjects. Drug Metab Dispos, 129:1748-54.

Pearson T, Denke M, McBride P, et al. 2004. Ezetimibe added to statin therapy reduces LDL-C and improves goal attainment in patients with hypercholesterolemia [abstract]. American College of Cardiology 53rd Annual Meeting, New Orleans, March 7-10, 2004.

Repa JJ, Mangelsdorf DJ. 2002. The liver X receptor gene team: potential new players in atherosclerosis. Nat Med, 8:1243-8.
Repa JJ, Turley SD, Lobaccaro JA, et al. 2000. Regulation of absorption and ABC1-mediated efflux of cholesterol by RXR heterodimers. Science, 289:1524-9.

Russell DW. 1999. Nuclear orphan receptors control cholesterol catabolism. Cell, 97:539-42.

Salen G, Tint GS, Shefer S, et al. 1999. Increased sitosterol absorption is offset by rapid elimination to prevent accumulation in heterozygotes with sitosterolemia. Arterioscler Thromb, 12:563-8

Seedorf U, Engel T, Lueken A, et al. 2004. Cholesterol absorption inhibitor Ezetimibe blocks uptake of oxidized LDL in human macrophages. Biochem Biophys Res Commun, 320:1337-41.

Shepherd J, Cobbe SM, Ford I, et al. 1995. Prevention of coronary heart disease with pravastatin in men with hypercholesterolemia. West of Scotland Coronary Prevention Study Group. N Engl J Med, 333:1301-7.

Shepherd J. 2004. Lipids in health and disease. Biochem Soc Trans, $32: 1051-6$.

Stamler J, Wentworth D, Neaton JD. 1986. Is relationship between serum cholesterol and risk of premature death from coronary heart disease continuous and graded? Findings in 356,222 primary screenees of the Multiple Risk Factor Intervention Trial (MRFIT). JAMA, 256:2823-8.

Steinberg D, Witztum JL. 1990. Lipoproteins and atherogenesis. Current concepts. JAMA, 264:3047-52.

Sviridov DD, Safonova IG, Pavlov MY, et al. 1990. Inhibition of cholesterol synthesis by lovastatin tested on six human cell types in vitro. Lipids, 25:177-9.

Van Heek M, Farley C, Compton DS, et al. 2000. Comparison of the activity and disposition of the novel cholesterol absorption inhibitor, SCH58235, and its glucuronide, SCH60663. Br J Pharmacol, 129:1748-54.

Wang DQH. 2007. Regulation of intestinal cholesterol absorbtion. Annu Rev Physiol, 69:221-48.

Wilson PW, Garrison RJ, Castelli WP, et al. 1980. Prevalence of coronary heart disease in the Framingham Offspring Study: role of lipoprotein cholesterols. Am J Cardiol, 46:649-54. 